\title{
CHANGES IN CLUSTERIN SERUM CONCENTRATION LEVELS IN ONCOLOGIC PATIENTS DURING THE COURSE OF SPA THERAPY - A PILOT STUDY
}

\author{
Ivan Vareka ${ }^{a, b *}$, David Stejskal ${ }^{c, d}$, Renata Varekova ${ }^{\mathrm{e}}$, Katerina Burianova ${ }^{\mathrm{b}}$, Jiri Hnatek $^{\mathrm{a}}$
}

a Spa Luhacovice Inc., Luhacovice, Czech Republic

${ }^{b}$ Department of Physiotherapy, Faculty of Physical Culture, Palacky University, Olomouc, Czech Republic

c Department of Laboratory Medicine \& Metabolic Out Patient Centre, Central Moravian Hospital, Prostejov, Czech Republic

d Department of Medical Chemistry and Biochemistry, Faculty of Medicine and Dentistry Palacky University, Olomouc, Czech Republic

e Department of Functional Anthropology and Physiology, Faculty of Physical Culture, Palacky University, Olomouc, Czech Republic

e-mail:IvanVareka@seznam.cz

Received: February 9, 2009; Accepted: May 8, 2009

Key words: Clusterin/Spa therapy/Cancer

Background: Clusterin (70-80 kDa; synonym ApoJ) is a stress-associated cytoprotective glycoprotein involved in many physiological and pathophysiological processes and it is up-regulated by various apoptotic triggers in many cancers and neurodegenerative diseases.

Aim: Measurement of serum clusterin values in individuals with a history of cancer before and after spa therapy.

Methods: Serum clusterin concentration (ELISA) was determined in a group of 26 oncologic patients ( 4 men and 22 women) at the beginning and at the end (the 18th or 19th day) of spa treatment. The spa treatment lasted 3 weeks. The patients with various types of cancer had undergone basic therapy (surgery, chemotherapy, actinotherapy) prior to spa treatment. They were divided according to the interval between the end of basic treatment and the start of spa therapy. Patients coming within 12 months comprised group A $(n=15)$ while patients coming later comprised group $\mathrm{B}(\mathrm{n}=11)$.

Results: clusterin concentrations increased in 11 patients (73\%) and decreased in $4(27 \%)$ in group A and increased in $5(45 \%)$ and decreased in $6(55 \%)$ in group B. The non-parametric sign test was non-significant. There were positive value of average change between the second and the first sample in group A and negative value in group B. In group A the parametric test showed significant increased clusterin concentration at the end of spa treatment but the data had non-parametric distribution in fact.

Conclusion: It is concluded that early spa therapy increases clusterin serum concentration. This is probably due to to the positive effects of balneotherapy. However the sample was very small and further research is required.

\section{INTRODUCTION}

In the Czech Republic, cancer patients are sent to spas for further complex treatment after basic therapy (surgery, actiontherapy, chemotherapy). The term "complex" means that the public health insurance pays for both the spa the treatment itself and accommodation and food. The recommendation to go to the spa is made by an oncologist. The basic complex spa therapy of oncologic patients lasted in this case 3 weeks and the patients underwent 1821 treatment procedures per week. The typical treatment programme in Luhacovice Spa consists of carbonated baths ( 3 times per week), conventional massage ( 3 times per week), water gymnastic (approx. 3 times per week) and other therapeutic procedures. The individual plan is prescribed by a spa physician on the basis of incoming physical examination, the physician takes into account both the basic diagnosis and other health problems as well as current health status. When clinically examined at the end of spa therapy, the patients showed an improvement both in mental and physical condition (joint mobility, cardio-pulmonary fitness, scar tissue release, etc.).

In recent years there has been increased demand for evidence-based medicine. This presents a problem for spa therapy owing to its complex nature as physical factors play an important role as do psychological issues. We seek a parameter that could be an index of complex spa therapy.

Clusterin (70-80 kDa; synonym ApoJ) is a stress-associated cytoprotective glycoprotein involved in a number of physiological and pathophysiological processes. This is up-regulated by various apoptotic triggers common to many cancers and neurodegenerative diseases. Two clusterin forms exist: the first (secretory, exogenous) occurs in biological fluids; the second form (endogenous) is contained in the cytoplasm and nucleus ${ }^{4}$. Secretory clusterin originates from preclusterin $(60 \mathrm{kDa})$, which is subsequently). This form has antiapoptotic $\beta$, $\alpha$ cleaved into two chains of mature protein (and antiangiogenetic effects. Endogenous clusterin originates in alternative cleaving of 
preclusterin; the final molecule acts as a strong stimulator of apoptosis ${ }^{2,3,5}$. Clusterin effects can be summarized as follows: secretory clusterin acts as a cytoprotective extracellular chaperon and its expression and synthesis are increased in individuals suffering from various forms of stress and especially in patients with malignant tumors ${ }^{1-5}$. Extracellular clusterin plays a number of roles, e.g. in the production of amyloid in Alzheimer's disease, binding abnormal prions, "heat shock proteins" and reduced degradation of many components of the extracellular matrix. Clusterin is also involved in immune regulation, lipid transport, sperm maturation and protection of cell membranes ${ }^{5}$. The results of experimental studies indicate the pathophysiologic importance of clusterin particularly in the pathogenesis of glomerulonephritis, polycystic kidneys, renal tubular diseases, neurodegenerative disorders, atherosclerosis, myocardial infarction and malignant tumors $^{5-6}$.

As the data are still sporadic and often contradictory, the aim of the present pilot study was to determine the changes in clusterin serum concentrations in oncologic patients who underwent a 3 week spa therapy.

\section{METHODS}

Thirty-one patients were initially selected for the research. They were sent to Luhacovice Spa after cancer diagnosis, only one (No.26) had bronchial asthma as her main diagnosis ( $\mathrm{J} 45.9$ ) but she was treated for pharynx carcinoma 18 months prior to the spa treatment.

The laboratory examination was done at the beginning (the 2nd or 3rd day) and at the end (the 18th or 19th day) of spa stay. Serum clusterin was determined by enzyme-linked immunosorbent assays (ELISA, Biovendor Research\&Development, Modrice, Czech Republic). Patients were acquainted with the aim of study. Twentysix patients ( 4 men and 22 women) gave blood samples and were selected into the final study group (Table 1). The basic anthropometric data (high, weight, BMI and fat $\%$ by impedance measurement) were measured in the course of incoming physical examination.

A one-way ANOVA for repeated measurement and post-hoc Fishers's LSD test were used as parametric tests, the sign test and Mann-Whitney test were used as nonparametric tests.

Table 1. Group characteristics and changes in clusterin serum concentration $(\mathrm{kU} / 1)$.

\begin{tabular}{|r|l|r|l|r|r|r|r|r|}
\hline No. & \multirow{2}{*}{ ICD-10 } & Gender & \multicolumn{1}{|c|}{ Dg. } & Age & S I & S II & Change & Int \\
\hline 1 & C 34.3 & W & lung ca & 74 & 46.8 & 53.1 & 6.3 & 3 \\
\hline 2 & C 53.8 & W & uterus ca & 74 & 51.3 & 45.6 & -5.7 & 24 \\
\hline 3 & C 90 & W & myeloma & 64 & 21.3 & 34.5 & 13.2 & 10 \\
\hline 4 & C 50.4 & W & mamma ca & 63 & 81.3 & 101.7 & 20.4 & 12 \\
\hline 5 & C 50.2 & W & mamma ca & 68 & 62.1 & 42.6 & -19.5 & 22 \\
\hline 6 & C 90 & W & myeloma & 73 & 46.8 & 50.1 & 3.3 & 5 \\
\hline 7 & C 80 & M & neck ca & 50 & 46.2 & 45 & -1.2 & 14 \\
\hline 8 & C 07 & M & ca parotis & 63 & 41.7 & 37.8 & -3.9 & 8 \\
\hline 9 & C 18 & W & caecum ca & 68 & 49.2 & 44.4 & -4.8 & 4 \\
\hline 10 & C 54.9 & W & uterus ca & 37 & 40.8 & 43.8 & 3 & 6 \\
\hline 11 & C 19 & W & rectum ca & 69 & 34.8 & 43.2 & 8.4 & 12 \\
\hline 12 & C 50.3 & W & mamma ca & 67 & 35.4 & 49.2 & 13.8 & 10 \\
\hline 13 & C 50.5 & W & mamma ca & 61 & 60.6 & 56.7 & -3.9 & 19 \\
\hline 14 & C 85.7 & W & lymfoma & 59 & 39.6 & 40.2 & 0.6 & 5 \\
\hline 15 & C 50 & W & mamma ca & 70 & 44.7 & 37.8 & -6.9 & 10 \\
\hline 16 & C 18 & W & lymfoma & 58 & 47.1 & 51.9 & 4.8 & 25 \\
\hline 17 & C 32.9 & M & larynx ca & 55 & 29.1 & 30.9 & 1.8 & 60 \\
\hline 18 & C 34.9 & M & lung ca & 64 & 39 & 42.6 & 3.6 & 26 \\
\hline 19 & C 56 & W & ovarian ca & 47 & 41.4 & 44.7 & 3.3 & 9 \\
\hline 20 & C 81 & W & M. Hodgkin & 39 & 37.2 & 41.4 & 4.2 & 120 \\
\hline 21 & C 54.1 & W & leiomyoma & 66 & 44.7 & 44.4 & -0.3 & 6 \\
\hline 22 & C 53.1 & W & uterus ca & 65 & 47.4 & 50.4 & 3 & 7 \\
\hline 23 & C 54.9 & W & uterus ca & 70 & 50.7 & 42.75 & -7.95 & 13 \\
\hline 24 & C 81.9 & W & M. Hodgkin & 41 & 45.6 & 42.9 & -2.7 & 39 \\
\hline 25 & C 18.3 & M & colon ca & 65 & 42.3 & 46.2 & 3.9 & 10 \\
\hline 26 & J 45.9 & W & pharynx ca & 61 & 36.9 & 40.2 & 3.3 & 18 \\
\hline
\end{tabular}

Explanations: No. - ordinal number, ICD-10 - diagnosis according to International Classification of Disease No. 10 , W - woman, M - man, Dg. - diagnosis, ca - cancer, S I(II) - sample I(II) of clusterin serum concentration (kU/1), Change - S II minus S I, Int - interval between the end of basic treatment and start of spa therapy (in months). 
Fig. 1. Changes in clusterin concentration in groups $\mathrm{A}$ and $\mathrm{B}$.

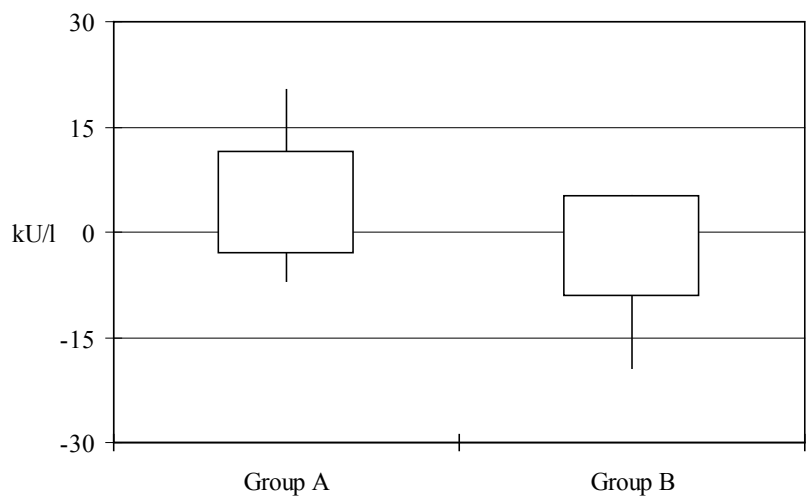

Explanations: the rectangular shows one standard deviation above and under the mean of difference between the first and second sample

\section{RESULTS}

The Table 1 provides basal characterization of study group.

The clusterin serum concentration was increased in 16 patients (62\%) and was decreased in 10 patients (38\%). Fig. 1 shows distinct difference between increasing clusterin concentration in group $\mathrm{A}$ at the end of spa stay (i. e. sample two minus sample one) and decreasing concentration in group B).

The changes were very small (Table 2, Fig. 1) and hence the significance was not set. Neither of the groups divided according to diagnosis, gender or age, height, weight, BMI or fat percent showed significant differences in changes of clusterin serum concentration.

When divided into group A (patients incoming within 12 months after basic therapy) and group B (incoming later), the clusterin concentration in group A increased in 11 patients $(73 \%)$ and decreased in $4(27 \%)$, in group B the concentration increased in $5(45 \%)$ and decreased in $6(55 \%)$. however the non-parametric sign test was non-significant.

Table 3 shows a positive value of average change between the second and the first sample in group A and

Table 2. Changes in clusterin concentration $(\mathrm{kU} / 1)$.

\begin{tabular}{|c|c|c|c|c|c|c|c|}
\hline & S I & S II & Change & t-test & \multicolumn{2}{|c|}{ Change } & sign test \\
\hline $\mathbf{n}$ & 26 & 26 & 26 & $\mathbf{p}$ & & $\mathbf{P}$ & $\mathbf{p}$ \\
\hline $\mathbf{M}$ & 44.77 & 46.31 & 1.54 & \multirow{2}{*}{0,326} & increased & $62 \%$ & \multirow{2}{*}{0,327} \\
\hline SD & 11.33 & 12.64 & 7.84 & & decreased & $38 \%$ & \\
\hline
\end{tabular}

Explanations: $\mathrm{n}$ - number, $\mathrm{M}$ - mean, SD - standard deviation, $\mathrm{p}$ - significance, for other see Table 1

Table 3. Patients divided according to time interval between the basic therapy and spa therapy.

\begin{tabular}{|c|c|c|c|c|c|c|c|c|}
\hline \multicolumn{4}{|c|}{ Group A (n=15) } & \multicolumn{4}{c|}{ Group B (n=11) } \\
\hline & A I & A II & Change & Int & B I & B II & Change & Int \\
\hline M & 43.88 & 48.1 & 4.22 & 7.8 & 45.98 & 43.87 & -2.11 & 34.55 \\
\hline SD & 12.43 & 15.71 & 7.39 & 2.79 & 10,09 & 6.53 & 7.2 & 29.86 \\
\hline
\end{tabular}

Explanation: A I(II)- sample I(II) of group A, B I(II) - sample I(II) of group B, for other see Tables 1 and 2

Table 4. Changes in clusterin concentration (kU/1) in Group A and B.

\begin{tabular}{|c|c|c|c|}
\hline & & & B II $(43,87)$ \\
\hline & & B I $(45,98)$ & 0,347 \\
\hline & A II $(48,1)$ & 0,663 & 0,521 \\
\hline A I $(43,88)$ & $\mathbf{0 , 0 3 5}{ }^{*}$ & 0,749 & 0,998 \\
\hline
\end{tabular}

Explanations: mean values in brackets, the others are values of significance; for the rest see previous tables. 
negative value in group B. Table 4 shows significantly higher clusterin concentration at the end of spa treatment in the group A, when ANOVA test for repeated measurement and post-hoc Fishers's LSD test was used.

\section{DISCUSSION}

Balneological procedures cause a particular physical response that increases self-defence factors of the body but this is induced in a very friendly and pleasant way. The higher stress level expresses itself probably as increase in clusterin serum concentration.

We noticed the clusterin concentration increased in patients who came to spa within 12 months after the end of basic treatment for malignant tumor. The differences were non-significant when the non-parametric test was used, only the parametric test was significant and showed a clear trend but the data had a non-parametric distribution in fact. Clusterin concentration in patients with longer than 12 months interval had a decreasing trend, however it was non-significant according to the non-parametric test as well as in the parametric test. One possible explanation is that the "early" patients had a more static way of life that was changed by the affirmative stress of the spa. The "later" patients had a more dynamic life style so that the complex spa treatment had a more soothing effect. This explanation is only a speculation due to small and non-consistent group observed in this pilot study.

In conclusion, the results are promising. It seems that early-initialised spa therapy is beneficial regarding the in- creased clusterin serum concentration that can by caused by affirmative stress as effect of balneotherapy. Later initialised spa therapy has a more soothing effect but the sample was very small and further research is warranted.

\section{ACKNOWLEDGEMENT}

The study has been supported by the research grant from the Ministry of Education, Youth and Sports of the Czech Republic (No. MSM 6198959221) "Physical Activity and Inactivity of the Inhabitants of the Czech Republic in the Context of Behavioral Changes".

\section{REFERENCES}

1. Gleave ME, Gleave J, Burt HM. The inhibition of angiogenesis by antisense oligonucleotides to clusterin. Angiogenesis 2005; 8: 229-8.

2. Kim BM, Kim SY, Lee S, Shin YJ, Min BH, Bendayan M et al. Clusterin induces differentiation of pancreatic duct cells into insulin-secreting cells. Diabetologia 2006; 49: 311-20.

3. Kim BM, Kim SY, Lee S, Shin YJ, Min BH, Bendayan M. et al. Clusterin induces differentiation of pancreatic duct cells into insulin-secreting cells. Diabetologia 2006; 49: 311-20.

4. Kruger S, Mahnken A, Kausch I, Feller AC. Value of clusterin immunoreactivity as a predictive factor in muscle-invasive urothelial bladder carcinoma. Urology 2006; 67: 105-9.

5. Silkensen J. Clusterin: physiologic and pathophysiologic considerations. Int J Biochem Cell Biol 1995; 27: 633-45.

6. Stejskal D, Vavroušková J, Mareš J, Urbánek K. Application of new laboratory marker assays in neurological diagnosis - a pilot study. Biomedical Papers 2005; 149: 265-267. 